\title{
PROLONGED HOSPITALISATION IN PATIENTS ADMITTED WITH ACUTE EXACERBATION OF COPD- PREVALENCE AND ITS PREDICTORS
}

\author{
Ronald Win $B^{1}$, Sindhu M. D2
}

${ }^{1}$ Additional Professor, Department of Pulmonary Medicine, Government Medical College, Trivandrum, Kerala. ${ }^{2}$ Senior Resident, Department of Pulmonary Medicine, Government Medical College, Trivandrum, Kerala.

\section{ABSTRACT}

\section{BACKGROUND}

Objectives

1. To study the prevalence of prolonged hospitalisation in patients admitted with acute exacerbation of COPD.

2. To study predictors of prolonged hospitalisation in patients admitted with acute exacerbation of COPD.

\section{MATERIALS AND METHODS}

Study Design- Cross sectional study.

Study Setting- Department of Pulmonary Medicine, Govt. Medical College, Thiruvananthapuram.

Study Period- March 2014 to August 2014.

Study Population- All patients admitted with acute exacerbation of COPD during March 2014 to August 2014.

Inclusion Criteria- Patients admitted with acute exacerbation of COPD.

Exclusion Criteria- Patients with asthma, asthma COPD overlap, those who were unwilling to participate were excluded.

Methodology- Baseline clinical data and investigations were recorded using structured proforma. COPD diagnosis was based on history and post-bronchodilator spirometry. Prolonged hospital stay defined as upper quartile of distribution of hospital stay. Factors contributing to prolonged hospitalisation were identified by case control analysis.

Data Analysis- SPSS software version 16.

\section{RESULTS}

Mean age was $66.5+$ /- 8.5 years. $85.4 \%$ were male. $22.9 \%$ of patients required prolonged hospitalisation. Identified predictors of prolonged hospitalisation were hypertension, diabetes, exacerbations during previous year, tachycardia, low saturation during admission, RBS $>200$ during admission.

\section{CONCLUSION}

Around one fourth of patients required prolonged hospital stay or admission to ICU. Apart from the general characteristics and severity of illness in individual patients comorbidities also contributed significantly to development of adverse outcomes.Previous exacerbation contributes significantly to prolonged hosptalsation, hence every effort should be taken to prevent exacerbations in COPD,and when it occurs should be managed promptly to prevent adverse outcomes.

\section{KEYWORDS}

COPD Exacerbation, Predictors, Prolonged Hospitalisation.

HOW TO CITE THIS ARTICLE: Win RB, Sindhu MD. Prolonged hospitalisation in patients admitted with acute exacerbation of COPD - Prevalence and its predictors. J. Evolution Med. Dent. Sci. 2017;6(18):1450-1454, DOI: 10.14260/Jemds/2017/316

\section{BACKGROUND}

COPD is a leading cause of morbidity and mortality worldwide and results in an economic and social burden that is both substantial and increasing. ${ }^{1}$ Exacerbations of COPD are important events in the course of disease because they negatively affect patient's quality of life, accelerate the rate of decline in lung function and are associated with significant mortality. In-hospital mortality of patients admitted for a hypercapnic exacerbation with acidosis is approximately $10 \%$. Mortality reaches $40 \%$ at 1 year after discharge in those needing mechanical support, and all-cause mortality 3 years after hospitalisation is as high as $49 \%$.

Financial or Other, Competing Interest: None.

Submission 19-01-2017, Peer Review 16-02-2017,

Acceptance 21-02-2017, Published 02-03-2017.

Corresponding Author:

Dr. Ronald Win B,

Additional Professor,

Department of Pulmonary Medicine,

Government Medical College, Trivandrum.

E-mail: ronaldwin_b@yahoo.co.in

DOI: $10.14260 /$ jemds $/ 2017 / 316$
COPD is associated with significant economic burden. In the European Union, the total direct costs of respiratory disease are estimated to be about $6 \%$ of the total health care budget, with COPD accounting for $56 \%$ of this cost of respiratory disease. In the United States, the estimated direct costs of COPD are $\$ 29.5$ billion and the indirect costs $\$ 20.4$ billion.

COPD exacerbations account for the greatest proportion of total COPD burden on the health care system. Hospitalisation for COPD exacerbations further increases the morbidity and economic burden. Early identification of patients with a prolonged stay due to acute exacerbation of chronic obstructive pulmonary disease (COPD) may reduce risk of adverse event and treatment costs. Prolonged hospitalisation can also cause adverse events like hospital acquired infection. Hence, the present study was planned with a primary objective to study the prevalence of prolonged hospitalisation for acute exacerbation of COPD and a secondary objective to find out the factors contributing to it. 


\section{MATERIALS AND METHODS}

This was a cross sectional study conducted in Department of Pulmonary Medicine, Govt. Medical College, Thiruvananthapuram. All patients admitted with acute exacerbation of COPD admitted under the department during March 2014 to August 2014 were included in the study. All patients with acute exacerbation of COPD, admitted in the Department of Pulmonary Medicine during the study period were selected. Baseline clinical data and investigations were recorded using structured proforma. COPD diagnosis was based on history and previous spirometry criteria according to GOLD guidelines. If a patient gets admitted more than once during the study period only first admission was taken for the study. Those patients not willing to give consent and cases of asthma and ACOS were excluded from the study. The criteria for admission and discharge were according to GOLD 2014.

AECOPD defined as worsening in patient's baseline dyspnoea, cough and or sputum production beyond the normal day-to-day variation lasting at least one day. There are no internationally accepted norms for optimum length of hospital stay for COPD exacerbation. Hence, prolonged hospital stay was defined as upper quartile of distribution of hospital stay in line with other studies.2-4 Quantitative variables were represented as arithmetic mean and standard deviation. Categorical variables were grouped and represented as percentage. Data was entered in excel sheet and analysed using SPSS version 16. Predictors of adverse outcome were identified by case control analysis, Odds ratio with $95 \%$ confidence intervals were calculated. A p value of $<0.05$ was taken as significant. Institution Research \& Ethics Committee clearance was obtained prior to the study.

\section{RESULTS}

144 patients were included in the study. Mean age of study population was $66.5+/-8.5$ years. $85.4 \%$ of patients were male, $13.9 \%$ of patients were non-smokers. $27.8 \%$ were current smokers. Rest of the patients were smokers who have already stopped smoking. Mean smoking index was $871.8+/$ 544.8. 80.6\% were having a smoking index more than 400.24 patients (16.9\%) had firewood smoke exposure.

Around $90 \%$ of the patients had either Grade 2 or Grade 3 MMRC dyspnoea in stable state. $51.4 \%$ patients presented within 7 days of increase in symptoms. All patients had increased dyspnoea on presentation. Increased cough and sputum production occurred in 129 patients. Fever was present in $34.7 \%$ and sputum purulence in $29.2 \%$ of patients. The main comorbidities were diabetes, hypertension and coronary artery disease. $27.1 \%$ were diabetic. $18.1 \%$ were hypertensive and $9 \%$ had coronary artery disease.

\section{General Characteristics of Patients}

\begin{tabular}{|c|c|}
\hline Variables & Number (Percentage) \\
\hline Increased dyspnoea & $144(100)$ \\
\hline Increased cough & $129(90)$ \\
\hline Increased sputum production & $129(90)$ \\
\hline Sputum Purulence & $42(29.2)$ \\
\hline Fever & $50(34.7)$ \\
\hline Pedal oedema & $39(27.1)$ \\
\hline Elevated JVP & $8(5.6)$ \\
\hline Arterial oxygen saturation $<92 \%$ & $66(44.1)$ \\
\hline Pulse rate $>100$ & $48(33.3)$ \\
\hline Diabetes & $20(13.9)$ \\
\hline Hypertension & $26(18.9)$ \\
\hline Coronary artery disease & $13(9)$ \\
\hline
\end{tabular}

Mean duration of hospital stay was $9.6 \pm 4.1$ days. Hospitalisation of 12 or more days was considered as a prolonged hospital stay, $22.9 \%$ were having prolonged hospital stay.

Patients with past history of hypertension, diabetes mellitus, previous history of exacerbations were found to have prolonged hospitalisation. Tachycardia at the time of admission, oxygen saturation of 92 or less at the time of admission, RBS $>200$ at the time of admission were associated with prolonged hospitalisation. There was no statistically significant difference between various GOLD stages and duration of hospitalisation, but there was statistically significant difference between combined COPD groups A, B, C \& D. Duration of hospital stay was more in groups B, C, and D compared to group A. Those patients with sputum culture and sensitivity yielding any organism were having prolonged hospitalisation than those without a sputum culture positivity and the association was statistically significant. There was no association between any of chest x-ray abnormalities and prolonged hospitalisation. There was no association between prolonged hospital stay and variables like age, sex, coronary artery disease, smoking status or elevated total count.

\section{Percentage distribution of the sample according to Age}

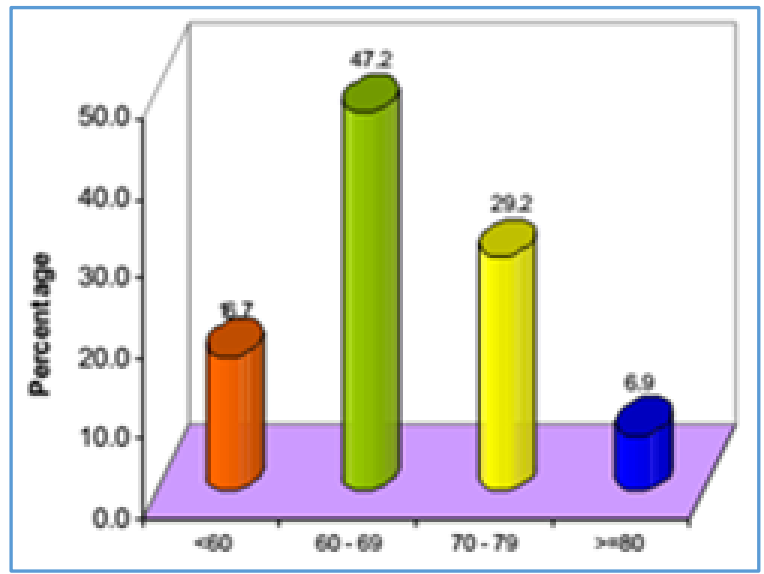

Figure 1

Mean age $66.5+/-8.5$ years. $47.2 \%$ of patients were between 60-69 years.

Percentage Distribution of the Sample According to Sex $\mathbf{8 5 . 4 \%}$ of Patients were Males

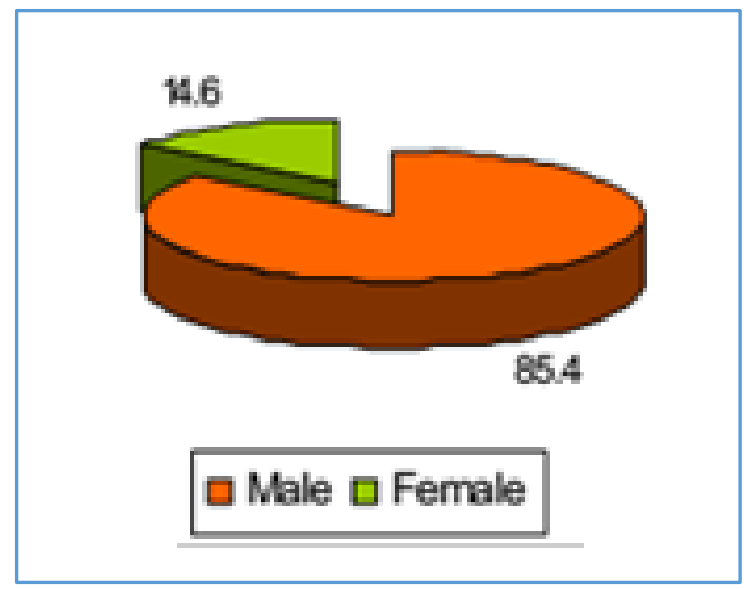

Figure 2 
Percentage Distribution of the Sample According to Smoking status

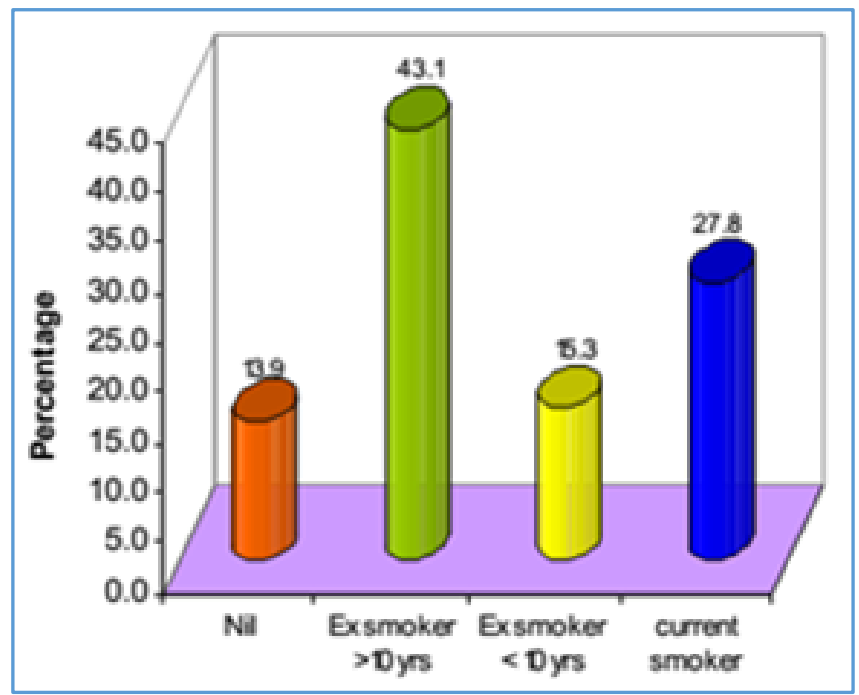

Figure 3

$13.9 \%$ of patients were non-smokers, $27.8 \%$ were current smokers. Rest of the patients were smokers who have already stopped smoking.

Percentage Distribution of the sample According to Smoking Index

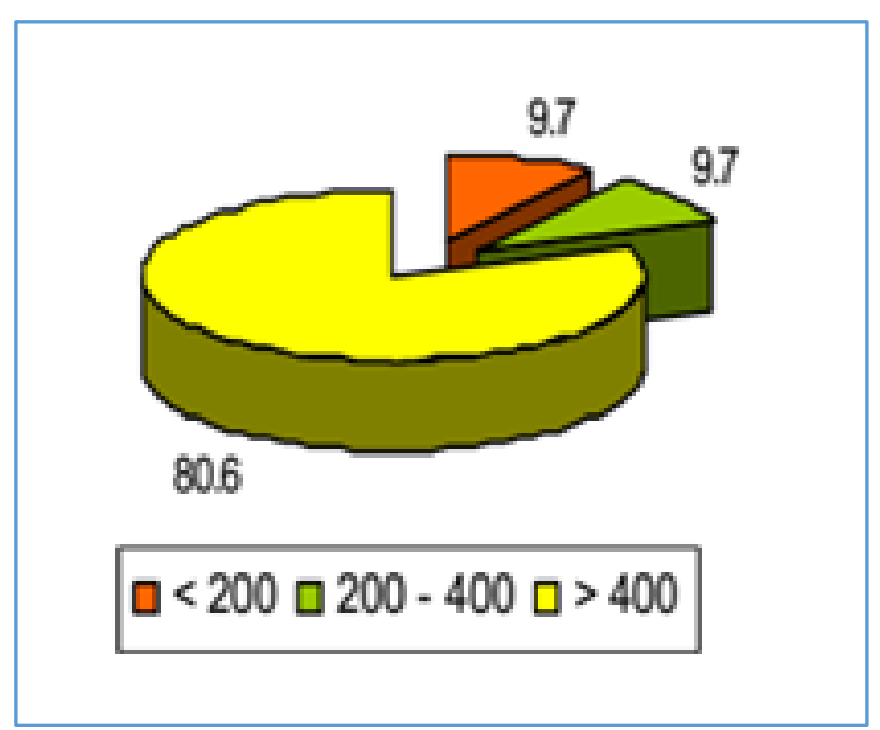

Figure 4

$80.6 \%$ were having a smoking index more than $400.9 .7 \%$ were having smoking index between 200-400 and $9.7 \%$ below 200. Mean smoking index was $871.8+$ +- 544.8 .
Percentage Distribution of Sample According to Number of Exacerbations in last year

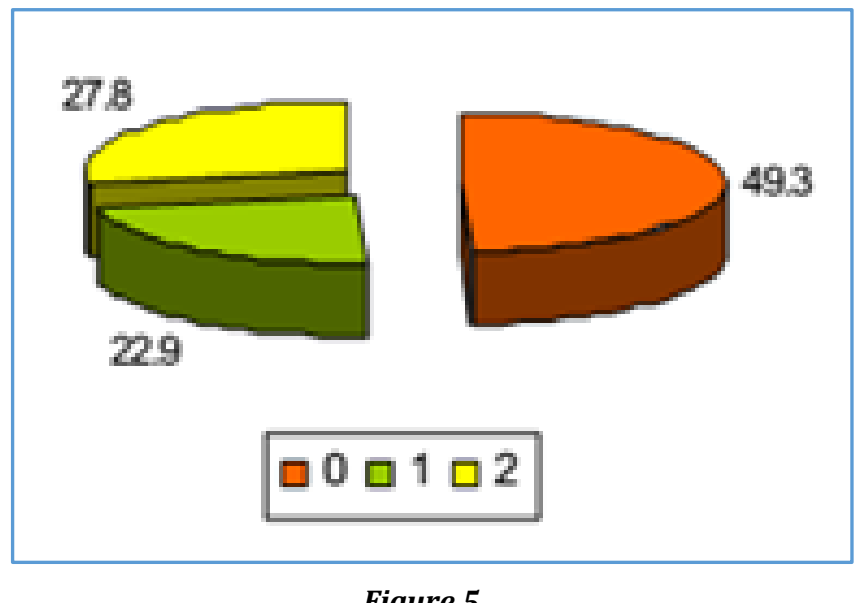

$49.3 \%$ of patients had no exacerbations in last year. $22.9 \%$ had one exacerbation during last year and $27.8 \%$ had 2 or more exacerbations in last year.

Percentage Distribution of the sample according to FEV1

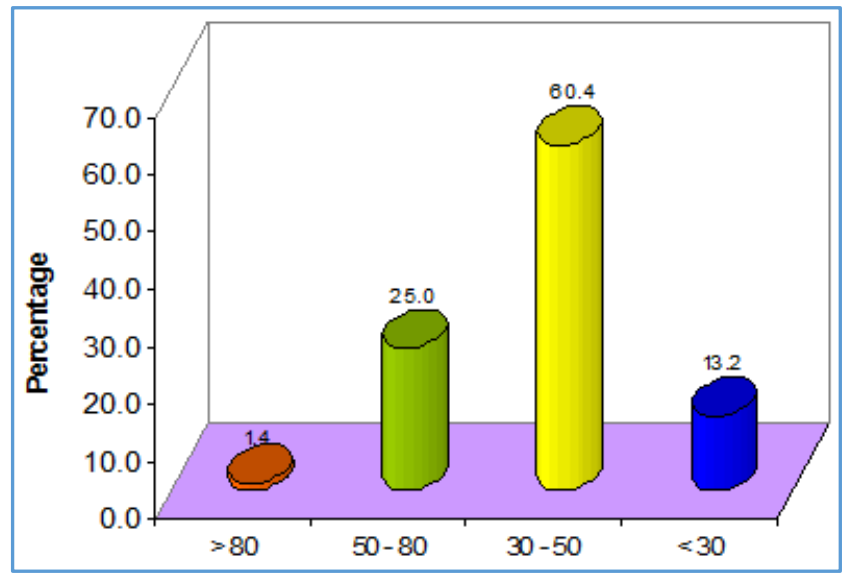

Figure 6

Percentage Distribution of the sample According to Combined COPD Assessment

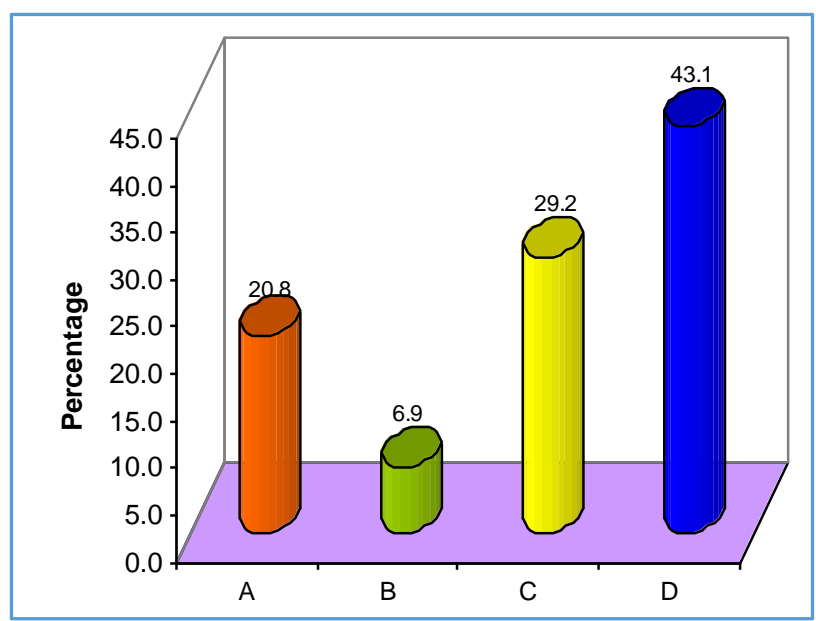

Figure 7 
Associations of Prolonged hospital stay and selected variables

\begin{tabular}{|c|c|c|c|c|c|c|c|}
\hline \multirow{2}{*}{ Variable } & & \multicolumn{2}{|c|}{$<=12$ days } & \multicolumn{2}{|c|}{$>12$ days } & \multirow{2}{*}{ Odds ratio } & \multirow{2}{*}{ P Vaue } \\
\hline & & Count & $\%$ & Count & $\%$ & & \\
\hline \multirow{3}{*}{ Sputum C\&S } & Nil & 31 & 91.2 & 3 & 8.8 & 1 & \multirow{3}{*}{$X^{2}=6.22^{*}, p=0.045$} \\
\hline & Sterile & 78 & 73.6 & 28 & 26.4 & 3.70 (1.05-13.09) & \\
\hline & $\begin{array}{c}\text { Organism } \\
\text { Detected }\end{array}$ & 2 & 50.0 & 2 & 50.0 & $10.33(1.05-102.08)$ & \\
\hline \multirow{2}{*}{ RBS } & $>200$ & 31 & 66.0 & 16 & 34.0 & $2.43(1.09-5.40)$ & \multirow{2}{*}{$\mathrm{X}^{2}=4.89^{*}, \mathrm{p}=0.027$} \\
\hline & $<=200$ & 80 & 82.5 & 17 & 17.5 & 1 & \\
\hline \multirow{4}{*}{ FEV1 } & $>80$ & 1 & 50.0 & 1 & 50.0 & $3.75(0.19-74.07)$ & \multirow{4}{*}{$X^{2}=9.4^{*}, p=0.024$} \\
\hline & $50-80$ & 34 & 94.4 & 2 & 5.6 & $0.22(0.04-1.34)$ & \\
\hline & $30-50$ & 61 & 70.1 & 26 & 29.9 & $1.60(0.48-5.28)$ & \\
\hline & $<30$ & 15 & 78.9 & 4 & 21.1 & 1 & \\
\hline \multirow{4}{*}{$\begin{array}{l}\text { Combined } \\
\text { COPD } \\
\text { Assessment }\end{array}$} & $\mathrm{A}$ & 29 & 96.7 & 1 & 3.3 & 1 & \multirow{4}{*}{$\mathrm{X}^{2}=18.43^{*}, \mathrm{p}=0.000$} \\
\hline & $\mathrm{B}$ & 7 & 70.0 & 3 & 30.0 & $12.43(1.12-138.23)$ & \\
\hline & $\mathrm{C}$ & 37 & 88.1 & 5 & 11.9 & $3.92(0.43-35.41)$ & \\
\hline & $\mathrm{D}$ & 38 & 61.3 & 24 & 38.7 & $18.32(2.34-143.40)$ & \\
\hline
\end{tabular}

\begin{tabular}{|c|c|c|c|c|c|c|c|}
\hline & & \multicolumn{4}{|c|}{ Hospital Stay } & \multirow{3}{*}{$\mathbf{Z}^{2}$} & \multirow{3}{*}{$\mathbf{P}$} \\
\hline & & \multicolumn{2}{|c|}{$<=12$} & \multicolumn{2}{|c|}{$>12$} & & \\
\hline & & Count & Percent & Count & Percent & & \\
\hline \multirow{2}{*}{ CAD } & Yes & 9 & 69.2 & 4 & 30.8 & \multirow[b]{2}{*}{0.5} & \multirow[b]{2}{*}{0.480} \\
\hline & No & 102 & 77.9 & 29 & 22.1 & & \\
\hline \multirow{4}{*}{ Smoking Status } & Nil & 16 & 80.0 & 4 & 20.0 & \multirow{4}{*}{0.58} & \multirow{4}{*}{0.900} \\
\hline & Ex-smoker $>10$ years & 46 & 74.2 & 16 & 25.8 & & \\
\hline & Ex-smoker $<10$ years & 17 & 77.3 & 5 & 22.7 & & \\
\hline & Current smoker & 32 & 80.0 & 8 & 20.0 & & \\
\hline \multirow{2}{*}{ Elevated Total count } & Yes & 13 & 65.0 & 7 & 35.0 & \multirow{2}{*}{1.92} & \multirow{2}{*}{0.166} \\
\hline & No & 98 & 79.0 & 26 & 21.0 & & \\
\hline
\end{tabular}

\section{Associations of Prolonged Hospital stay and Selected Variables}

\begin{tabular}{|c|c|c|c|c|c|}
\hline & & $<=12$ Days & >12 Days & & \\
\hline Variable & & $\%$ & $\%$ & Odds ratio & P Value \\
\hline \multirow{2}{*}{ HTN } & Yes & 61.5 & 38.5 & $2.58(1.04-6.43)$ & \multirow{2}{*}{$\mathrm{X}^{2}=4.34^{*}, \mathrm{p}=0.037$} \\
\hline & No & 80.5 & 19.5 & 1 & \\
\hline \multirow{2}{*}{$\mathrm{DM}$} & Yes & 53.8 & 46.2 & $5.14(2.23-11.84)$ & \multirow{2}{*}{$\mathrm{X}^{2}=16.35^{* *}, \mathrm{p}<0.001$} \\
\hline & No & 85.7 & 14.3 & 1 & \\
\hline \multirow{3}{*}{ Exacerbations } & 0 & 90.1 & 9.9 & 1 & \multirow{3}{*}{$\mathrm{X}^{2}=13.7^{* *}, \mathrm{p}=0.001$} \\
\hline & 1 & 66.7 & 33.3 & $4.57(1.58-13.25)$ & \\
\hline & 2 & 62.5 & 37.5 & $5.49(2.0-15.05)$ & \\
\hline \multirow{2}{*}{ Pulse rate } & $>100$ & 56.3 & 43.8 & $5.44(2.37-12.50)$ & \multirow{2}{*}{$X^{2}=17.69^{* *}, p=0.000$} \\
\hline & $<=100$ & 87.5 & 12.5 & 1 & \\
\hline SPO2 & $<=92$ & 66.7 & 33.3 & $2.83(1.26-6.35)$ & $\mathrm{p}=0.010$ \\
\hline
\end{tabular}

\section{DISCUSSION}

The study was a hospital based cross sectional study, conducted in the Department of Pulmonary Medicine, Medical College, Thiruvananthapuram. 144 patients admitted with acute exacerbation of COPD were recruited. It was observed that $47.2 \%$ patients were aged between 60 and 70 years, and mean age of presentation was $66.5 \pm 8.5$ years. Mean age was lower than previous studies. In the study done by Wakae Hasegwa et al, mean age was 77.5 years ${ }^{5}$ and in similar study by Zinka Matkovic et al mean age of presentation was $70.0 \pm$ 9.5. 6

Out of 144 patients, 123 were male (85.4\%) and 21 patients were female $(14.6 \%)$. This was comparable with study by Zinka Matkovic et al in which $84 \%$ were male and $16 \%$ were female.6 Proportion of female patients were around $50 \%$ in various other studies of Ying Wang et al, Yan Ching et al. 7,8 This difference may be due to the differences in smoking habits between the population. Around $90 \%$ of the patients had either Grade 2 or Grade 3 MMRC dyspnoea in stable state. $51.4 \%$ patients presented within 7 days of increase in symptoms. All patients had increased dyspnoea on presentation. Increased cough and sputum production occurred in 129 patients. Fever was present in $34.7 \%$ and sputum purulence in $29.2 \%$ of patients.

The main comorbidities were diabetes, hypertension and coronary artery disease. $27.1 \%$ were diabetic. $18.1 \%$ were hypertensive and $9 \%$ had coronary artery disease. Previous studies show prevalence of diabetics in COPD patients between $10-25 \%$ and $32.5-55 \%$ for hypertension. ${ }^{9}$ The present study group had a lower mean age compared to many previous studies, which may be the cause for decreased number of hypertensives. Out of 144 patients, $34(23.6 \%)$ patients required ICU admission. 17 patients $(11.8 \%)$ required non-invasive ventilator support. $2.8 \%$ required invasive mechanical support. 8 patients (5.6\%) expired during hospital stay. 
Mean FEV1 of study group was $47.1 \pm 13.9$. Around $85.4 \%$ patients were in either GOLD stage II/III. According to combined COPD assessment, 43.1\% were in group D and $29.2 \%$ in Group C. Mean pulse rate at presentations was 95.2 \pm 10.1 . This was comparable with study by Ian G Shell et al. 10 Features of cardiac failure was present in $5.6 \%$ patients and arterial oxygen saturation was less than $92 \%$ in $44.1 \%$. Total counts were elevated more than 11,000 in $13.9 \%$ patients. Sputum culture and sensitivity yielded organisms in 4 patients and it was sterile in $73.6 \%$. RBS at the time of admission was more than 200 in $32.6 \%$.

Mean duration of hospital stay was $9.6 \pm 4.1$ days. Hospital stay $>12$ days is taken as prolonged hospital stay. Similarly in a study by Ying Wang et al, mean duration of hospital stay was defined by hospital stay more than 11 days.7 In study by Zinka Matkovitc, mean duration of hospitalisation was $8.8 \pm 5.5$ days, while 11 days was established as cut-off to define prolonged hospitalisation. ${ }^{6}$ 22.9 percent of patients in the present study had prolonged hospital stay. Similar to this, prolonged hospital stay was $21 \%$ in the study by Zinka Matkovitc et al.

Factors associated with prolonged hospital stay identified in the present study were hypertension, diabetes, hyperglycaemia i.e. RBS $>200 \mathrm{mg} \%$, number of exacerbations last year, pulse rate $>100 /$ minute at the time of admission, arterial oxygen saturation $<92 \%$ at the time of admission, and combined COPD group D compared to group A or B. A study by Ying Wang7et al and Parapill11 et al identified diabetes as a factor associated with prolonged length of hospital stay. Similar to the present study, there was no association between prolonged hospital stay and variables like age, sex, FEV1, and X-ray findings of pneumonia. Roberts, Lowe, Bucknall et al described age, performance status and FEV1 as independent predictors of hospital stay. 12

\section{CONCLUSION}

Around one fourth of patients required prolonged hospital stay or admission to ICU. Apart from the general characteristics and severity of illness in individual patients, comorbidities also contributed significantly to development of adverse outcomes. Previous exacerbation contributes significantly to prolonged hospitalisation, hence every effort should be taken to prevent exacerbations in COPD, and when occurs it should be managed promptly to prevent adverse outcomes.

\section{REFERENCES}

[1] Lopez AD, Shibuya K, Rao C, et al. Chronic obstructive pulmonary disease: current burden and future projections. Eur Respir J 2006;27(2):397-412.

[2] Incalzi RA, Pedone C, Onder G, et al. Predicting length of stay of older patients with exacerbated chronic obstructive pulmonary disease. Aging (Milano) 2001;13(1):49-57.

[3] BuSaba NY, Schaumberg DA. Predictors of prolonged length of stay after major elective head and neck surgery. Laryngoscope 2007;117(10):1756-63.

[4] McDevitt J, Kelly M, Comber H, et al. A populationbased study of hospital length of stay and emergency readmission following surgery for non-small-cell lung cancer. Eur J Cardiothorac Surg 2013;44(4):e253-9.

[5] Hasegawa W, Yamauchi Y, Yasunaga H, et al. Factors affecting mortality following emergency admission for chronic obstructive pulmonary disease. BMC Pulm Med 2014;14:151.

[6] Matkovic Z, Huerta A, Soler N, et al. Predictors of adverse outcome in patients hospitalised for exacerbation of chronic obstructive pulmonary disease. Respiration 2012;84(1):17-26.

[7] Wang Y, Stavem K, Dahl FA, et al. Factors associated with a prolonged length of stay after acute exacerbation of chronic obstructive pulmonary disease (AECOPD). Int J COPD 2014; 9:99-105.

[8] Cheng Y, Borrego ME, Frost FJ, et al. Predictors for mortality in hospitalized patients with chronic obstructive pulmonary disease. Springer plus 2014;3:359.

[9] Smith MC, Wrobel JP. Epidemiology and clinical impact of major comorbidities in patients with COPD. Int J COPD 2014;9:871-88.

[10] Stiell IG, Clement CM, Aaron SD, et al. Clinical characteristics associated with adverse events in patients with exacerbation of chronic obstructive pulmonary disease: a prospective cohort study. CMAJ 2014;186(6):E193-204.

[11] Parappil A, Depczynski B, Collett P, et al. Effect of comorbid diabetes on length of stay and risk of death in patients admitted with acute exacerbations of COPD. Respirology 2010;15(6):918-22.

[12] Roberts C, Lowe D, Bucknall C, et al. Clinical audit indicators of outcome following admission to hospital with acute exacerbation of chronic obstructive pulmonary disease. Thorax 2002;57(2):137-41. 